Universidad de Lima

Facultad de Psicología

Carrera de Psicología

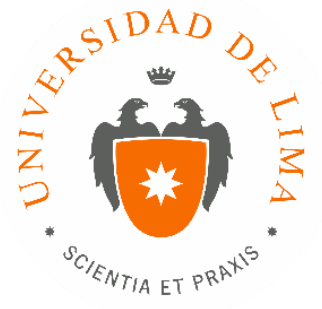

\title{
DEFINICIÓN Y DISEÑO DEL MODELO DE EVALUACIÓN DE DESEMPEÑO EN UNA EMPRESA DEL RUBRO TECNOLOGÍA
}

Trabajo de suficiencia profesional para optar el título profesional de Licenciado en Psicología

\author{
Camila Arellano Huerta
}

Código 20112582

Lima - Perú

Febrero de 2019 


\section{DEFINICIÓN Y DISEÑO DEL MODELO DE EVALUACIÓN DE DESEMPEÑO EN UNA EMPRESA DEL RUBRO TECNOLOGÍA}




\section{TABLA DE CONTENIDO}

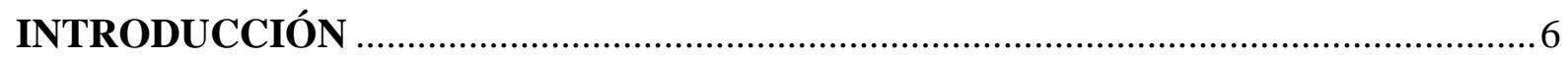

CAPÍTULO I: IDENTIFICACIÓN DEL PROBLEMA ................................................ 7

CAPÍTULO II: DESCRIPCIÓN DE LAS ACTIVIDADES REALIZADAS ................ 10

2.1 Definición del Modelo de Evaluación de desempeño.................................................... 10

2.2 Diseño de la Evaluación de desempeño.................................................................. 11

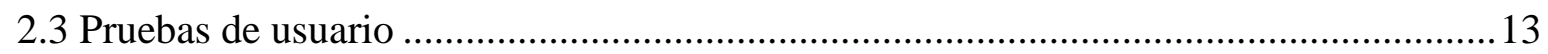

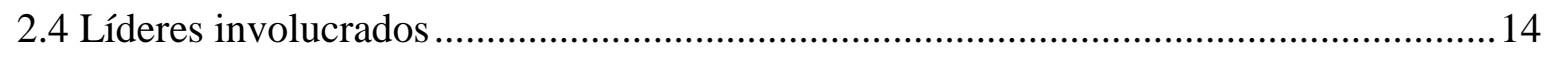

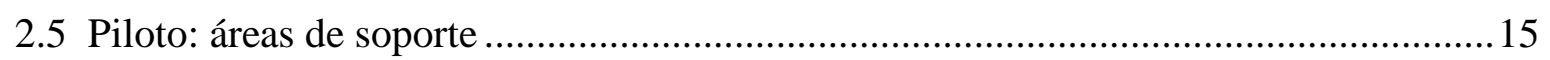

CAPÍTULO III: RESULTADOS DE LA INTERVENCIÓN......................................... 16

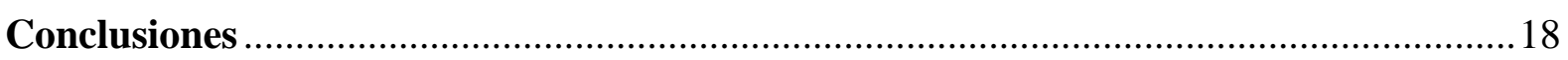

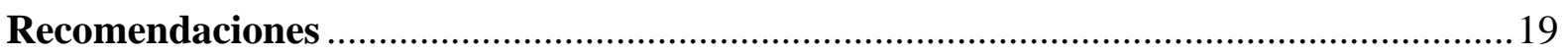

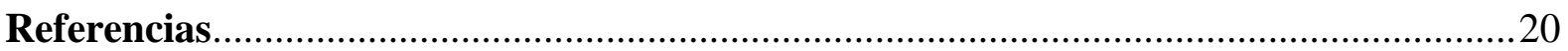




\section{ÍNDICE DE FIGURAS}

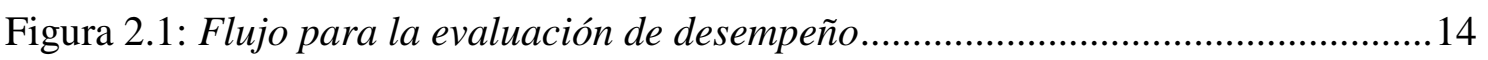




\section{INTRODUCCIÓN}

La evaluación de desempeño es una herramienta que permite medir el rendimiento de los colaboradores y el valor que agrega a los resultados del negocio; es decir, medir la ventaja competitiva de la gente en contribución al negocio (Rojas, 2015). Así mismo, según Research at Great Place To Work (2016), la evaluación de desempeño es un procedimiento por el que se miden y evalúan las habilidades y los resultados de un colaborador, con el objeto de establecer su productividad y fijar medidas para su rendimiento futuro. Por lo tanto, esta tiene como objetivos principales medir el potencial de cada colaborador, buscar las posibilidades de crecimiento personal teniendo en cuenta los objetivos de la empresa y los del personal trabajador y potenciar a los colaboradores como un elemento básico de toda la empresa. El impacto de la evaluación de desempeño en la gestión de los recursos humanos en las organizaciones incide en distintos aspectos; como en la optimización de la selección de personal, detección de áreas de mejora y el establecimiento de bases para el desarrollo de una carrera profesional. Además, realizar evaluaciones de desempeño periódicamente puede llevar a la implementación de cambios en la empresa que favorezcan la productividad y la motivación de los colaboradores.

De esta manera, se observa cómo la evaluación de desempeño es un proceso clave para la gestión de los recursos humanos en las organizaciones; es por esto que, el presente trabajo describe el proyecto de definición y diseño del modelo de evaluación de desempeño en una empresa del sector de servicios de tecnología peruana. Este proyecto abarca desde la definición del modelo, pasando por distintas etapas que concluye con la prueba piloto de la herramienta sistematizada de evaluación de desempeño diseñada como parte de este nuevo modelo, a un grupo específico dentro de la organización. 


\section{CAPÍTULO I: IDENTIFICACIÓN DEL PROBLEMA}

La empresa sobre la cual se hará referencia en este trabajo es de capital peruano, pertenece al rubro de servicios de tecnología, cuenta actualmente con 1400 colaboradores y con 35 años en el mercado; sin embargo, desde el 2015 empezó un proceso de transformación debido a que pasó a manos de un nuevo grupo inversionista, separándose del grupo al que pertenecía inicialmente. Esto trajo consigo muchos cambios, que van desde una nueva razón social y nombre comercial de la compañía hasta un proceso de transformación en los procesos internos de la misma, los cuales se encuentran en constante cambio hasta el día de hoy.

Dentro de estos procesos, están aquellos que pertenecen al área de Gestión Humana, más específicamente, el proceso de Evaluación de Desempeño, el cual es el foco de este trabajo.

La evaluación de desempeño en el Perú, muestra diversos resultados. Un estudio realizado por PriceWaterhouseCooper (citado en Montoya, 2016) en el 2014, que incluyó a 115 importantes empresas de once sectores, indica que para ese año un $64 \%$ de empresas peruanas ya contaba con un proceso formal de evaluación de desempeño, un $14 \%$ se encontraba en proceso de implementación del mismo, el 13\% consideraba tener una evaluación a corto plazo y solo un $9 \%$ no contaba con un proceso formal de evaluación de desempeño. Además, la evaluación de desempeño se encontraba como el tercer proceso de recursos humanos más desarrollado por las empresas; siendo reclutamiento y selección el proceso más desarrollado y capacitación y desarrollo el segundo. Por otro lado, las empresas encuestadas utilizaban la gestión de desempeño como input para capacitar y desarrollar a sus colaboradores y, en segundo lugar, para determinar ascensos y promociones.

Ahora, la evaluación de desempeño en la empresa en la cual se enfoca este trabajo no era un proceso que se encontraba implementado ni se encontraba en desarrollo; es decir, era parte de ese porcentaje que no contaba con un proceso formal de este proceso. Durante el 2017, los 
Gerentes y Jefes de la organización realizaron diversas solicitudes a Gestión Humana para poder medir el rendimiento de sus equipos de una manera objetiva, pues querían saber cuáles eran las brechas de sus equipos y, además, buscaban darles retroalimentación teniendo un “input” objetivo el cual los respaldara. Hasta ese momento, la compañía no contaba con ninguna herramienta que permitiera hacer frente a las necesidades de los líderes en cuanto a mediciones de desempeño. Por otro lado, una auditoria a inicios del 2018 por parte de un cliente muy importante para la organización solicitaba tener un modelo de evaluación de desempeño establecido. Debido a que no contar con este modelo, suponía poner en riesgo el contrato con este cliente, Gestión Humana decidió plantear una evaluación de desempeño con la cual se pudiera cubrir esta brecha a corto plazo. Sin embargo, esta evaluación consistió en la elaboración de una base simple en MS Excel, con la cual se pudiera medir de manera rápida el desempeño de los colaboradores. Ya que esto fue creado únicamente para la auditoria de este cliente, el desempeño fue medido a los colaboradores que trabajaban con el mismo (20 colaboradores). Una vez la auditoría finalizó, este modelo no se replicó al resto de la empresa, pues contaba con muchas deficiencias y fue creado únicamente para poder hacer frente al problema presentado de manera rápida.

Tras haber pasado por esta situación, en la que la empresa pudo notar cómo no contar con un modelo de evaluación de desempeño formal pudo haber sido un factor para la no renovación del contrato con uno de sus principales clientes, la creación de un nuevo modelo de evaluación de desempeño se convirtió en una de las prioridades para el área de Formación y Desarrollo de Gestión Humana.

Ahora, debido a la situación financiera por la cual la empresa se encontraba pasando, no era factible poder obtener alguna herramienta que ya existiera en el mercado; por lo cual, este nuevo modelo de evaluación de desempeño debía ser desarrollado por la misma organización. Al tratarse de una empresa del rubro tecnológico, fue posible poder desarrollar esta nueva 
herramienta de Evaluación de Desempeño "inhouse" en conjunto con el equipo del software de Recursos Humanos, el cual se encuentra dentro del portafolio comercial de la compañía. A partir de esto, en un trabajo conjunto entre el área de Formación y Desarrollo y el equipo del software de Recursos Humanos se pasó a definir el nuevo modelo, diseñar una herramienta de evaluación de desempeño sistematizada que respondiera a la realidad de la empresa y realizar un piloto en las áreas de soporte con el fin de probar la herramienta y medir el nivel de adopción de ésta por los colaboradores para en un futuro, poder replicarla en toda la organización. 


\section{CAPÍTULO II: DESCRIPCIÓN DE LAS ACTIVIDADES Y TAREAS REALIZADAS}

\subsection{Definición del Modelo de Evaluación de desempeño}

Para poder definir cuál sería el modelo de desempeño y poder realizar la herramienta de evaluación en el Software de RRHH, se realizó en primer lugar, un benchmark teniendo en cuenta las principales herramientas de evaluación de desempeño que existen en el mercado. Así, se revisaron herramientas como SAP SuccessFactors y Acsendo Flex con el fin investigar cómo evalúan el desempeño estas herramientas y cuál es la metodología que usan.

Así mismo, se revisó diversas fuentes acerca de los métodos que estas herramientas usan. Se encontraron distintos métodos de evaluación de desempeño y se optó por usar el método de evaluación con base en el futuro, referido a evaluar el potencial del colaborador y el establecimiento de objetivos mediante autoevaluaciones y administración de objetivos, con el cual se busca convertir los objetivos organizacionales en los objetivos individuales. Esto hace que los colaboradores tengan más probabilidades de alcanzar los objetivos, con lo cual, la motivación aumenta (Werther y Davis, 2008). Así mismo, se buscó realizar una evaluación de desempeño de $90^{\circ}$; en el cual el jefe directo evalúa al colaborador, pues al realizar un análisis de la población se identificó que; al ser la primera vez que se implementaba un modelo de desempeño formal en una herramienta sistematizada, este debía ser lo más simple posible para que los colaboradores puedan adaptarse rápidamente. Sin embargo, se buscó que los trabajadores puedan ser parte de esta evaluación; es por esto que, el modelo establecido para la compañía contiene una autoevaluación, la cual tiene como objetivo que los colaboradores sean parte de su 
evaluación de desempeño y que se pueda contar con una comparación entre las evaluaciones de los jefes y colaboradores.

En el modelo planteado la evaluación de desempeño consta de la evaluación tanto de objetivos y de competencias, de los cuales el $80 \%$ de la evaluación corresponde a la medición de objetivos y el $20 \%$ a la de competencias. Es importante mencionar que la autoevaluación no contiene un porcentaje asignado en la evaluación de desempeño final.

El modelo creado es dinámico, con cual se buscó que, en un futuro y con una organización más madura, pueda repotenciarse y llegar a ser un modelo de $360^{\circ}$.

\subsection{Diseño de la Evaluación de desempeño}

Una vez el modelo estuvo definido, se pasó al diseño de la herramienta. Como se mencionó previamente, se buscó desde un principio realizar la evaluación de desempeño de manera sistematizada, pues se consideró importante enlazar la tecnología con la gestión de personas y el rendimiento laboral. Al ser una empresa de tecnología, se contaba con los recursos necesarios para poder desarrollar el módulo de evaluación de desempeño dentro de un software de Recursos Humanos “inhouse”. Se realizó una primera reunión con el equipo de este Software en dónde se explicó la necesidad que se tenía para contar con una evaluación de desempeño sistematizada y solicitar el apoyo necesario. A partir de aquí, se creó un Gantt de trabajo, en el cual se establecieron fechas, entregables y avances para el desarrollo de la herramienta.

Una parte importante dentro del diseño de la herramienta fue la definición de las competencias organizacionales que se evaluarían en la evaluación de desempeño y el establecimiento del flujo que la herramienta debía seguir. Para lo primero; si 
bien es cierto, la empresa ya contaba con competencias organizacionales, estas no estaban definidas por niveles. Por esto, se realizó la definición de las competencias según los tres niveles de posiciones que existen en la empresa: estratégico, táctico y operativo. Una vez las competencias estuvieron definidas por el área de Formación y Desarrollo, se pasó a la validación de las mismas por la Gerencia de Gestión Humana y posteriormente, Gerencia General.

En relación al flujo que debía seguir la herramienta, se tomó en cuenta el "benchmark" de las herramientas de evaluación de desempeño que existen en el mercado para poder tener información y una guía para plantear el flujo. Así, se definió que la evaluación y; por tanto, la herramienta contaría de dos fases: definición de objetivos y evaluación. Se buscó desde un principio que los colaboradores pudieran ser parte de la evaluación; es por esto que, además de poder autoevaluarse tanto en el cumplimiento de objetivos como de competencias, se definió que los colaboradores que ocupan puestos estratégicos y tácticos podrían definir sus propios objetivos, los cuales debían responder a los objetivos del área que eran previamente establecidos por las gerencias, utilizando la metodología SMART; con la cual los objetivos deben seguir los siguientes criterios: ser específicos, pues se definen los resultados específicos a alcanzar; medibles, pues se definen cantidades, costos o métricas de calidad que determinan el progreso del objetivo; alcanzables porque se definen objetivos desafiantes pero realizables; relevantes, pues contribuyen al logro de las metas del equipo o de la organización y; por último, deben ser limitados en el tiempo; es decir, especificar una fecha de entrega, un margen de tiempo o frecuencia (Byham y Wellins, 2017). A continuación, se presenta el flujo definido para la evaluación de desempeño: 


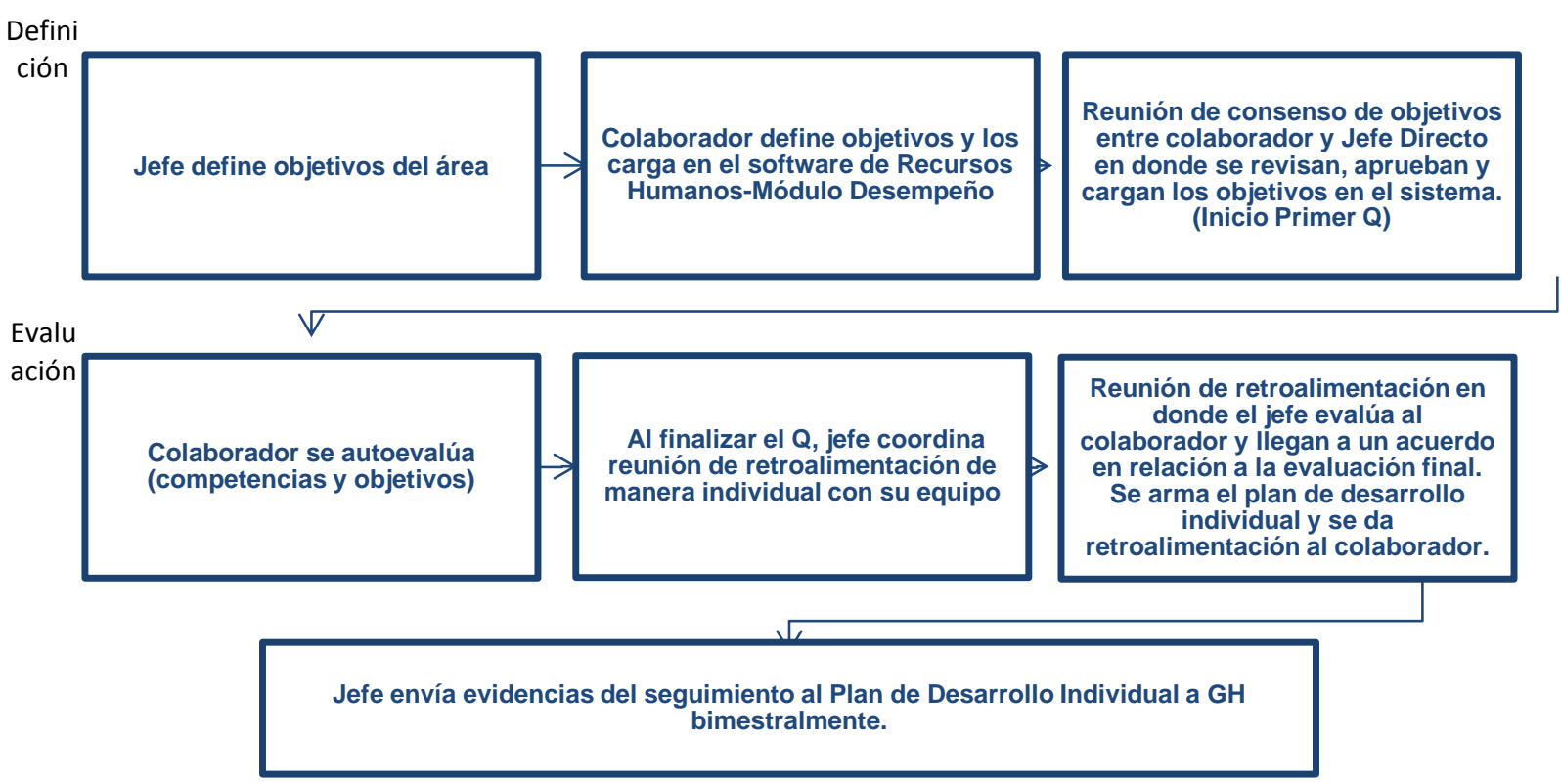

Figura 2.1. Flujo para la evaluación de desempeño

\subsection{Pruebas de usuario}

Una vez que la herramienta de evaluación de desempeño estuvo diseñada, se pasó a realizar pruebas de usuario con dos áreas: selección del personal y administración del personal. Estas pruebas tenían como objetivo identificar aquellos aspectos que debían ser cambiados, comprobar si la herramienta era amigable para el usuario y si el flujo que se había creado era el adecuado. Para realizar las pruebas de usuario, se diseñó un taller dictado por el área de Formación y Desarrollo en conjunto con el Jefe de Proyecto del equipo del software de Recursos Humanos., en el cual se explicó en qué consistía el modelo de evaluación de desempeño en desarrollado, los beneficios que traería tener una evaluación de desempeño formal y sistematizada, el flujo del proceso, cómo definir objetivos SMART y; por último, la parte práctica del taller, en la cual se presentó la herramienta desarrollada y se 
realizaron simulaciones tanto de la fase de Definición de objetivos como la de Evaluación.

\subsection{Líderes involucrados}

Una vez realizados los cambios necesarios en el modelo de evaluación de desempeño y validarlo con la Gerencia de Gestión Humana, se involucró a los líderes de la organización. Para esto, se realizaron reuniones con ellos explicándoles en qué consistía el nuevo modelo de evaluación de desempeño, las ventajas que traería contar con este nuevo modelo, el cual implicaba una medición más objetiva de sus equipos en base a objetivos propuestos por ellos mismos y competencias. Dentro de los beneficios presentados a los líderes estuvieron que ahora la organización contaría con una metodología consistente para el establecimiento de metas, monitoreo, revisión formal de desempeño, identificación de fortalezas, brechas y desarrollo de habilidades necesarias. Así mismo, se podría identificar a colaboradores claves y desarrollar el plan de carrera de los mismos; otra ventaja presentada fue que se podrán realizar mejores programas de gestión humana como los de reconocimiento y retención (Romero, 2015). Estas reuniones tuvieron como objetivo principal fidelizar al líder con el nuevo modelo de evaluación de desempeño antes de iniciar con el piloto que se iba a realizar. Para esto; además de las explicaciones que se dieron sobre el modelo, se presentó de manera rápida la herramienta diseñada y se resolvieron todas las dudas que los líderes pudieran tener al respecto. 


\subsection{Piloto: áreas de soporte}

Como cierre de este proyecto, se llevó a cabo un piloto de la evaluación de desempeño sistematizada con las áreas de soporte de la organización, las cuales están conformadas por un total de 200 colaboradores. Si bien es cierto, la evaluación consta de dos fases (definición de objetivos y evaluación), el piloto fue realizado únicamente de la primera fase. Para esto, se reunió a los líderes de los distintos equipos con el fin de contextualizarlos en el proceso y generar compromiso hacia este nuevo proceso, además de enseñarles el funcionamiento de la herramienta y la metodología para definir los objetivos del área. Así mismo, se trabajó de la mano con el área de Comunicación Interna, con el fin de realizar una campaña que soporte el lanzamiento de este nuevo proceso de evaluación de desempeño en las áreas que formaron parte de este piloto. Una vez iniciado el proceso, se realizaron reuniones con los distintos equipos para capacitarlos en la herramienta y presentarles los objetivos del área que habían sido definidos previamente con los líderes. Una vez los equipos habían sido capacitados en el uso de la herramienta, se estableció un periodo de tiempo para cargar los objetivos en el sistema y realizar las reuniones de consenso entre Jefe Directo- Colaborador; al mismo tiempo que el área de Formación y Desarrollo realizaba el seguimiento a los equipos participantes para lograr la mayor cantidad de objetivos aprobados y cargados en la herramienta.

Al día de hoy, la primera etapa ha sido concluida y se empezará con la siguiente etapa a mitad de este año para concluir todo el proceso de evaluación de desempeño. 


\section{CAPÍTULO III: RESULTADOS DE LA INTERVENCIÓN}

La medición de este proyecto se realizó una vez concluida la prueba piloto bajo el indicador de nivel de adopción de la herramienta de evaluación de desempeño. Para esto, la meta establecida fue lograr el $70 \%$ de objetivos cargados en el sistema una vez concluida la fase de definición de objetivos. Los resultados indicaron que se logró un $71.5 \%$ de objetivos cargados, lo que equivale a 143 personas de las 200 que conformaron la prueba piloto. Se estableció lograr un $70 \%$ de objetivos cargados, pues se tomó en cuenta que era la primera vez que los colaboradores pasaban por un proceso de evaluación de desempeño y, además, lo hacían dentro de una herramienta sistematizada.

Por otro lado, al finalizar la etapa de pruebas de usuario, los resultados mostraron que se debía ajustar el flujo de la evaluación, pues los usuarios manifestaron que no tenían claridad sobre cuáles eran las etapas del proceso y en qué partes debían participar y en cuáles no; es por esto que, se decidió establecer dos fases claras en todo el proceso: definición de objetivos y evaluación. Así mismo, los usuarios sugirieron realizar cambios en las definiciones de las competencias organizacionales a evaluar, ya que estas eran muy largas y les tomaba mucho tiempo poder leerlas, entenderlas y evaluarlas; lo cual, generaba mayor tiempo en poder finalizar tanto las autoevaluaciones como las evaluaciones de competencias. Así, con este input, se revisaron las definiciones de las competencias y se re definieron para hacer el proceso de evaluación más ágil.

Así mismo, como resultado del proyecto, ahora la organización cuenta con un modelo de evaluación de desempeño establecido, el cual incluye una herramienta sistematizada adaptada a la realidad de la empresa. Con esta evaluación de desempeño se tendrá una medición más objetiva; con lo cual, se podrán llevar a cabo mejores planes de capacitación y desarrollo para los colaboradores. Así, contar con una evaluación de desempeño formal impacta de manera 
directa en el rendimiento futuro de los colaboradores, pues la evaluación nos brinda información real sobre las brechas que existen en el desempeño de los colaboradores y en base a eso, permitirá crear planes de acción que ayuden a cerrar estas brechas y lograr colaboradores con un mejor desempeño. Además, con ayuda de este nuevo modelo de evaluación de desempeño, estas brechas estarán enfocadas no solo en aspectos técnicos sino también en las competencias que deberán ser potenciadas en los colaboradores; trabajando así, por el desarrollo y bienestar de estos durante el tiempo que permanezcan en la compañía. Por otro lado, la estrategia empleada en el proyecto consistió en primer lugar, levantar las necesidades que tenía la organización en relación a la falta de una evaluación de desempeño formal, en segundo lugar, se evaluó la situación y se definió los pasos a seguir, los cuales consistieron en organizar el proyecto fijando tiempos, responsables y tareas específicas. Además, fue de vital importancia la comunicación constante entre el área de Formación y Desarrollo y el equipo del software de Recursos Humanos en el cual se desarrolló la herramienta sistematizada. Por último, el mantener involucrado al Gerente de Gestión Humana y; posteriormente, involucrar a los líderes de la organización fue un aspecto clave que garantizó el éxito del proyecto y el cumplimiento del indicador (nivel de adopción de la herramienta) pues al estar los líderes comprometidos con el proceso, el seguimiento y cargue de los objetivos en la herramienta fue más rápido.

Cabe recalcar que, en relación a la aceptación del modelo de evaluación de desempeño diseñado, este tuvo una buena aceptación tanto por parte de los líderes como de los colaboradores; lo cual se puede comprobar con el cumplimiento del indicador establecido. 


\section{CONCLUSIONES}

A continuación, se presentan las conclusiones del proyecto:

- Involucrar a los líderes durante el proyecto, hacer parte a los colaboradores de su propia evaluación de desempeño durante la definición de objetivos y la autoevaluación, realizar las pruebas de usuario, en donde se pudieron identificar aquellos aspectos que debían ser cambiados en la herramienta antes de realizar la prueba piloto, fueron algunos de los motivos por los cuales se logró el cumplimiento del meta relacionado al modelo de evaluación de desempeño desarrollado.

- Contar con un modelo de evaluación de desempeño formal en esta organización brindará información necesaria para la correcta gestión del recurso humano de la empresa. Así, este es un "input" importante para poder realizar planes de capacitación y desarrollo, planes de sucesión y línea de carrera. De la misma manera, motiva a los colaboradores, pues se puede detectar si hay colaboradores que se encuentran en puestos en los que no están desarrollando su potencial al máximo y tomar acción de esto; por ejemplo, buscar otro puesto más adecuado en el que el colaborador pueda desarrollar una carrera profesional.

- Evaluar el contexto y la realidad por la que se encontraba pasando la organización antes de realizar el modelo de evaluación de desempeño contribuyó a lograr el nivel de adopción de la herramienta deseado. Esto, debido a que la herramienta respondía a las necesidades de la empresa teniendo en cuenta la audiencia a la que se dirigía. 


\section{RECOMENDACIONES}

A continuación, se detallarán las recomendaciones:

- Dada la influencia que tienen los líderes sobre los colaboradores, se recomienda mantener involucrados a los líderes durante todo el proceso de desarrollo de un nuevo modelo de evaluación de desempeño en la organización, pues son el principal soporte para que los equipos puedan responder de la manera esperada ante los cambios. Además, sería relevante que estos fomenten espacios de comunicación en sus equipos en donde puedan realizar seguimiento al cumplimiento de objetivos planteados en la evaluación de desempeño.

- Al ser la información que brinda la evaluación de desempeño un input importante para la gestión de los recursos humanos de la compañía, se recomienda designar a personas responsables que realicen el consolidado de los resultados de las evaluaciones de desempeño de la empresa y crear una matriz que permita acceder a la información de manera rápida. Esto, con el fin de poder trabajar la data proporcionada de manera más eficiente y usarla como "input" para los planes que se diseñen en el futuro en materia de gestión humana.

- Para futuros proyectos similares se recomienda evaluar el contexto organizacional; de esta manera, se podrán desarrollar herramientas que hagan frente a las necesidades de la compañía y se adapten a la realidad de la misma. 


\section{REFERENCIAS}

Byham, T. M., y Wellins, R. S. (2017). Tu primer trabajo como líder. Cómo un líder catalizador obtiene lo mejor de los demás. Buenos Aires: Ediciones Granica.

Montoya, D. (2016). Relación entre el clima organizacional y la evaluación del desempeño del personal en una empresa de servicios turísticos: Caso PTS Perú 2015 (Tesis de maestría). Recuperado de http://tesis.pucp.edu.pe/repositorio/handle/123456789/7490

Research at Great Place to Work. (2016). La importancia de la evaluación del desempeño para las empresas. Recuperado de http://www.greatplacetowork.com.py/publicaciones-y-eventos/blogs-y-noticias/884-la-importancia-de-la-evaluacion-del-desempeno-para-las-empresas

Rojas, M. (2015). Evaluación de Desempeño. España: Universidad Católica de Cordova.

Romero, R. A. (2015). Reflexión Crítica de la Administración del Desempeño. Revista Publicando, 2(1), 35-51. Recuperado de https://rmlconsultores.com/revista/index.php/crv/article/view/21/pdf_15

Werther, W., y Davis K. (2008). Administración de recursos humanos. El capital humano de las empresas (6ta ed.). México: McGraw-Hill/ Interamericana Editores, S.A. 